\title{
Comparison of patients' needs for information on prostate surgery with printed materials provided by surgeons
}

\author{
Philip Meredith, Mark Emberton, Carol Wood, Jackie Smith
}

\begin{abstract}
Objectives-To identify strengths, weaknesses, and omissions in existing leaflets and factsheets on prostatectomy given by surgeons to patients.

Design-Comparison of content of leaflets and factsheets with patients' needs and discontents in a questionnaire survey as part of the national prostatectomy audit. Setting-All NHS and independent hospitals performing prostatectomy in four health regions.

Subjects -87 surgeons, 53 of whom used printed material to inform patients about their operations; a total of 25 different factsheets being used. 5361 men undergoing prostatectomy were sent a closed response questionnaire about their treatment; 4226 men returned it completed. A random sample of 2000 patients was asked for further comments, of whom 807 supplied pertinent comments.
\end{abstract}

Main measures-Content of the 25 factsheets compared with patients' needs identified in the questionnaires.

Results-Much of the information distributed had considerable shortcomings: it lacked uniformity in form and content, topics of relevance to patients were omitted, terminology was often poor, and patients' experience was at variance with what their surgeons said. For example, only one factsheet discussed the potential consequences of malignancy. Patients wanted more information on prostate cancer $(1250(29 \%))$ and some thought that the explanation of biopsy results was inadequate $(29(4 \%))$. Only six factsheets discussed the possible changes in sexual sensation after transurethral resection of the prostate, stating that patients would feel no change. However, 1490(35\%) patients reported a change and $500(12 \%)$ were worried about it.

Surgical Audit Unit, Royal College of Surgeons of England, London WC2A 3PN Philip Meredith, senior research fellow

Mark Emberton, research fellow

Carol Wood, research assistant

Jackie Smith, research assistant

Correspondence to: Dr Meredith

Accepted for publication 31 October 1994 standards of prin information do not meet the needs and requirements of patients undergoing prostatectomy.

(Quality in Health Care 1995;4:18-23)

Keywords: patient information, patients' needs, prostate surgery.

\section{Introduction}

Studies have shown that whenever patients are given the opportunity to ask for information about their medical treatments they choose to do so. ${ }^{1-3}$ For such information to be useful, however, it must address the needs of the patient. Information should be concise, unambiguous, and given at the right time. ${ }^{4}$ One of the attributes of printed material as an adjunct to oral information is that it allows patients to assimilate information at their own pace and can be referred to later. ${ }^{5}$

Through the national prostatectomy audit ${ }^{6}$ we had access to the printed information given by surgeons to patients undergoing prostatectomy in four British health regions. We asked patients to assess the quality and usefulness of the information offered to them before and after admission to hospital. Patients also had the opportunity to expand on their closed response questions with free text comments. We compared the printed information about transurethral resection of the prostate given by surgeons to patients with the information needs expressed by the patients themselves.

\section{Methods}

Surgeon factsheets - Printed information materials, leaflets or factsheets, were gathered by contacting all surgeons participating in the national prostatectomy audit who used printed material as part of their information package for patients. We analysed the content of the factsheets and the presentational style of the leaflets.

Information from patients - A questionnaire was posted to all patients (5361 men) participating in the national prostatectomy audit. From this we were able to use data from a number of closed response questions which were directly relevant to information needs (box). In addition, a subsample of 2000 patients was randomly selected to obtain a further insight into information requirements by analysing the free text comments included in the questionnaire responses. These written comments were used to complement the statistical patient data when identifying patient needs and discontents.

Topics - From these two sources of information we were able to create a list of topics that we thought might be included in an ideal booklet.

Analysis of individual topics - We assessed each factsheet in terms of whether information had been given on the topics on our list. A topic was considered to have been properly addressed if sufficient detail was given to make the patient aware of the issue. Two researchers compared totals and reconsidered any borderline cases when deciding independently 


\section{Closed response questions from the national prostatectomy audit}

Q1 Please show us by ticking the boxes, what extra information would have been helpful to you with regard to your recent experience of surgery. You may answer more than one.

$\square$ About possible side effects $\square$ About the catheter
$\square$ About alternatives to surgery $\quad \square$ About the first weeks at home $\quad \square$ About tests I had to have
Q2 Did you have any difficulties getting erections (keeping the penis hard) before the operation?
$\square$ Not at all
Q3 Have you had any difficulties getting or keeping erections since the operation?
$\square$ Not at all
Q4 Has the sensation of ejaculation changed since the operation?
$\square$ Not at all
Q5 How much are you worried by any change in your sexual function since the operation?
$\square$ Not at all $\quad \square$ Some of the time
$\quad \square$ A little

whether a topic was to be included. Patients' comments were included in the results only when they had identified a topic during the analysis.

\section{Results}

SURGEON FACTSHEETS

Overall, $61 \%(53 / 87)$ of surgeons indicated that they gave printed information to patients preoperatively. We received copies of this information from 33 of the 53 surgeons $(62 \%)$. Once duplicated sheets (several surgeons used identical sheets) had been removed 25 unique factsheets remained, and these formed the basis of the study. They ranged in style from professionally printed brochures (six) to typeset black and white pages (three) and pieces of A4 paper (16), some of which were photocopied to near illegibility. Occasional typographical errors and ill formed sentences added to the homemade character of some examples. Five of the factsheets used fonts that were so small that many elderly patients would have difficulty in reading them. However, the presentation of the material seemed not to be related to the quality of the content.

\section{PATIENTS}

A total of $4226(79 \%)$ men returned completed questionnaires. Of the sample of 2000 patients, $807(40 \%)$ had added pertinent written comments, $296(37 \%)$ of which were critical of some aspect of the surgical service.

TOPICS

Seventeen topics were identified as being the most important areas for inclusion in a patient information booklet or factsheet. Table 1 lists them and the number of factsheets covering them.

ANALYSIS OF INDIVIDUAL TOPICS

Physiology

The location of the prostate was described by 22 of the 25 factsheets; nine also provided a diagram, which ranged from a home produced outline of relevant organs to commercially produced cut away imagery. Twenty one sheets described the prostate gland, although only one used the accurate description of "a fleshy organ ... made of glands and muscle." Five of these sheets gave an analogy to size ranging from "a small orange," "doughnut," and "apple with the core removed" to "walnut" and "almond." Fourteen gave some explanation of prostatic function. The uncertainty about the true function of the prostate was reflected in the range of functions accorded to it. For example:

"It secretes a small quantity of fluid for nourishing sperms."

"The prostate produces most of the ejaculated fluid."

"It helps with the passage of sperm."

"[It is a] sperm supercharger."

\section{Consequences of prostate enlargement}

Nineteen of the factsheets described the process of prostate enlargement as a normal development, with seven of them implying that normal development itself produces problematic constriction. The malfunctioning bladder

Table 1 List of topics relating to prostatectomy and numbers of factsheets including each topic

\begin{tabular}{|c|c|}
\hline Topic & $\begin{array}{c}\text { No of } \\
\text { factsheets } \\
(n=25)\end{array}$ \\
\hline \multicolumn{2}{|l|}{ Physiology: } \\
\hline Location & 22 \\
\hline Description & 21 \\
\hline Function & 14 \\
\hline Consequences of enlargement & 19 \\
\hline Symptoms and clinical severity & 14 \\
\hline Cancer & 6 \\
\hline Clinical tests & 4 \\
\hline \multicolumn{2}{|l|}{ Choice of treatment: } \\
\hline $\begin{array}{l}\text { Transurethral resection of the prostate/open } \\
\text { surgery only }\end{array}$ & 20 \\
\hline Other methods & 4 \\
\hline $\begin{array}{l}\text { Description of transurethral resection of the } \\
\text { prostate }\end{array}$ & 24 \\
\hline Hospital stay & 10 \\
\hline Catheter & 24 \\
\hline Outcome & 14 \\
\hline Adverse effects & 4 \\
\hline $\begin{array}{l}\text { Waiting time for transurethral resection of the } \\
\text { prostate }\end{array}$ & 1 \\
\hline Postoperative pain management & 14 \\
\hline Recovery & 17 \\
\hline Return to activity & 18 \\
\hline \multicolumn{2}{|l|}{ Sexual intercourse: } \\
\hline Some general mention & 23 \\
\hline Retrogade ejaculation & 11 \\
\hline Potency & 17 \\
\hline Sensation & 6 \\
\hline Follow up & 19 \\
\hline
\end{tabular}


as a consequence of enlargement was mentioned in 20 sheets. The choice of language used when describing prostate enlargement, though surgically correct, was often insensitive to the lay meaning of tumour as indicative of malignancy. For example:

"... around the age of 40 the prostate... can start to show signs of ageing, usually by the development of a benign tumour. These tumours are as common as grey hair."

Symptoms and their relation to clinical severity Inconsistency in defining the symptoms associated with prostate enlargement was evident. Fourteen sheets referred to symptoms consistent with bladder outflow obstruction. Of these, one sheet mentioned the possibility of renal failure resulting from prostatic obstruction and 10 mentioned acute urinary retention. Non-obstructive symptoms - for example, the "jumpy bladder" - were discussed in two of these 14 factsheets. Only three of them tried to discuss symptoms that had other causes.

\section{Cancer}

Cancer was addressed in six factsheets. In these sheets the weighting of the information from the point of view of patient confidence varied greatly. For example:

\begin{abstract}
"In only a small number of men there is a cancerous growth of the prostate."

"You will be told if your prostatic tumour was malignant."

"Occasionally the laboratory reports indicate that your prostate gland has a growth which would regrow fairly quickly. Further tests, operations and possible $x$ ray treatment would then be discussed with you."
\end{abstract}

Only one factsheet discussed the potential consequences of malignancy.

Nearly a third of respondents (1250/4226 $(30 \%)$ ) wanted more information on prostate cancer. Furthermore 29 of the men (4\%) adding free text comments criticised the inadequate information given to them about their biopsy results.

\section{Clinical tests}

Four factsheets mentioned tests that might be needed to determine the nature and seriousness of symptoms.

Over a tenth of respondents (611(14\%)) indicated that they needed more information on diagnostic tests. In addition, 29(4\%) patients writing free text comments criticised the poor procedures for informing them about details and results of both preoperative and postoperative tests.

\section{Choice of treatment}

Surgery was discussed in 22 sheets as though it was both inevitable and always effective in resolving symptoms. When considering surgical treatment most of them (20) discussed the choice as being limited to either transurethral resection of the prostate or open surgery. Only four referred to the use of other methods such as microwaves, lasers, or stents. Two out of these four also referred to patient participation in deciding about non-surgical interventions.

Over a quarter of respondents $(1085(26 \%))$ wanted information on treatment options other than transurethral resection of the prostate.

\section{Description of surgery (transurethral resection of} the prostate)

Twenty four factsheets included a brief description of transurethral resection of the prostate. The clarity of language was variable:

“... 'core' out the enlarged gland with an instrument passed 'up' or 'through' the penis."

"The prostate is removed via the penis." "[The prostate is] removed by an electrical current (diathermy) through the natural passage."

Only 3 of the 24 mentioned the use of an electric knife or current to remove the tissue.

A diagram was included in nine of the 24 factsheets as an aid to the written description, but the style was always anatomical rather than pictorial and could be regarded as confusing rather than clarifying.

\section{Stay in hospital}

Fewer than half (10) of the factsheets referred to the practicalities of being in hospital - for example, admission procedures, preparation for the operating theatre, and discharge.

\section{Catheters}

Twenty four of the factsheets mentioned the need for a catheter but only one of them described the experience of having a catheter in place and only one provided a diagram. Three factsheets referred to the importance of keeping the catheter clean.

A fifth of respondents $(894(21 \%))$ indicated that they would have liked more information about the catheter.

\section{Outcome}

Fourteen factsheets discussed the likely outcome of the operation as alleviating all symptoms. The 11 others omitted any mention of outcome and gave the impression that symptoms would be totally relieved.

\section{Adverse effects}

The possibility of non-resolution of critical symptoms or worsening of symptoms as a result of surgery was addressed by four factsheets. Among the subjects discussed were incontinence and irritable bladder. Only one factsheet mentioned the possibility of death in elderly men undergoing transurethral resection of the prostate.

Waiting for transurethral resection of the prostate One factsheet informed patients that their general practitioner could request an earlier 
admission to hospital than the date given if their symptoms worsened. None of the sheets provided any indication of the likely waiting period for admission.

A few of the free text comments $(17(2 \%))$ focused specifically on the unacceptably long wait for admission, given the patients' degree of pain or discomfort. Many patients appeared disturbed by the prolonged use of catheters before admission.

\section{Postoperative pain management}

The possibility of postoperative pain was mentioned in 14 factsheets, though there were clear differences in emphasis and wording. For example:

“.. burning and stinging when passing water."

“.. discomfort on removal of catheter."

"... burning sensation from the raw wound and/or infection."

"Patients should ask for pain killers rather than suffer in pain."

Over a tenth of respondents (105(13\%)) expressed dissatisfaction with the lack of information on postoperative pain. Many patients who had been given some information felt that the severity and duration of the pain had been underestimated.

\section{Recovery}

Estimates of the timing for total recovery varied significantly from six weeks to three months for complete recovery. Most factsheets (17) also described what the patient could expect during the recovery period. One factsheet referred to the continued risk of urinary incontinence and another mentioned the likelihood of having postoperative bladder scanning to ensure that voiding was complete. Phrasing and the use of probability in conveying the recovery process varied widely. For example:

"There will be 'slight discomfort' for $\mathrm{N}$ weeks."

"Most men ... are surprised just how little discomfort is involved."

"... symptoms improve rapidly."

Nearly a third of respondents (1195(28\%)) would have found it helpful to have more information about coping during the first weeks at home after the operation. Furthermore, 1551 of them $(37 \%)$ said that they would have liked extra information about side effects. Ninety five of those who enclosed written comments reported the lack of improvement in their symptoms after surgery. Most of these also recounted a range of unwanted effects resulting from the operation. In addition, many were uncertain about what was a "normal" consequence of the operation and what was an unusual or unfortunate side effect and how long such symptoms were likely to persist. A further 40 patients expressed dissatisfaction with the recovery information they were given. In general, these respondents stated that the information they had been given considerably underestimated the time it would take for symptoms to subside, if such information was given at all.

\section{Return to activity}

Eighteen of the factsheets discussed the everyday activities that might be attempted during recovery, but these guidelines varied considerably. For example:

"You should not drive a car for 3 weeks."

"You may start driving a car after a few days."

"You should be able to get back to normal activities by 3 weeks."

\section{Sexual intercourse}

The effect of transurethral resection of the prostate on a patient's sex life was described to some extent by 23 of the factsheets. Three important areas of sexual function were covered: retrograde ejaculation, impotence, and changes in sensation. For each of these topics, however, a wide range of expressions and sometimes confusing terminology was used.

Twenty three factsheets discussed retrograde ejaculation using various phrases. For example:

“... semen backfires."

"You will not emit any semen from your penis."

"You will probably not emit any semen from your penis."

"The sperm will ... go into the bladder."

The effects of retrograde ejaculation on fertility were mentioned by 11 sheets, but the terminology was confusing. For example:

\section{"You are unlikely to produce any children following this operation." \\ "Fertility is very much reduced but absolute sterility is not guaranteed." \\ "If you have not completed your family it is important to inform the doctor."}

Potency was addressed in 17 factsheets, although only six of them mentioned the possibility of the operation causing impotence in some cases. The remaining 11 indicated that no change in potency would occur as a result of this operation. Thus factsheets were contradictory. For example:

\footnotetext{
"Potency should not be affected by this operation."

"Erections cannot be avoided ... and will do no harm."

"A prostate operation may cause impotence in a $\%$ of patients."

"A few patients do experience inability to get a satisfactory erection."
}

Table 2 shows that $32 \%$ of men $(415 / 1320)$ who had no difficulties getting an erection before the operation had experienced problems 
Table 2 Erectile difficulties before and after surgery. Values are numbers (percentages) for 3392 respondents

\begin{tabular}{lccc}
\hline & \multicolumn{3}{c}{ After surgery } \\
\cline { 2 - 4 } & Not at all & $\begin{array}{c}\text { Some of } \\
\text { the time }\end{array}$ & $\begin{array}{c}\text { All of the } \\
\text { time }\end{array}$ \\
\hline Before surgery: & & & \\
Not at all & $905(68)$ & $246(19)$ & $169(13)$ \\
Some of the time & $151(14)$ & $599(56)$ & $313(30)$ \\
All of the time & $31(3)$ & $79(8)$ & $899(89)$ \\
\hline
\end{tabular}

since the operation. In contrast, $3 \%$ of men (31/1009) who had experienced problems before the operation no longer had a problem afterwards $(\mathrm{P}<0.0001)$.

With regard to changes in sexual sensation, six factsheets discussed this issue, all of them stating that the operation would create no change in the sensation of orgasm. For example:

"The sensation of ejaculation is maintained."

"There is no change in your erections or climax."

"You should continue to have the sensation of an orgasm."

The response to the national prostatectomy audit questionnaire seemed to contradict the impression given by the factsheets: $996(24 \%)$ patients reported a constant change of sensation and a further $494(12 \%)$ reported an occasional change. Overall, 189 patients $(5 \%)$ indicated that they were very worried by the change in their sexual function after the operation, and a further $311(7 \%)$ were quite worried. They did not specify, however, which aspect of sexual function was a problem.

\section{Follow up}

Some guidance on follow up care was given in 19 factsheets. The follow up outpatient visit was discussed in 12 of the 19 sheets, two of them mentioning the need to take a urine sample to the meeting. Five of the factsheets specifically referred the patient to either the ward or their general practitioner if they were troubled by any further problems.

\section{Discussion}

These results indicate a mismatch in the information currently being given to patients undergoing prostatectomy and the information that patients need. Furthermore, in many topics the information was in direct conflict with the experiences reported by patients. This was especially evident in respect of sexual activity.

The content of the factsheets was not uniform, and evidence supported the findings of the Audit Commission that clinical staff were producing leaflets based on their own rather than their patients' knowledge. ${ }^{5}$ Our surgeons scored highly on including topics of surgical importance, such as describing transurethral resection of the prostate and the function and description of the prostate, but they omitted more general topics of concern to patients such as the hospital experience, side effects, and options other than surgery. When detailing information on topics such as recovery there was little standardisation and the guidelines varied considerably. Information may be formulated anecdotally, however, because so little reliable evidence exists on these aspects of the procedure.

Omissions in information on such issues as cancer and test procedures may well reflect traditional surgical wisdom: do not alarm patients by telling them details they do not need to know or what is "unlikely" to affect them. The questionnaire showed, however, that these topics are important to patients and that they want information to gain a better idea of all the possible as well as probable implications of their condition and treatment. Of course some omissions in information may have been covered in the outpatient meeting, although the time constraints in most outpatient clinics make it hard to believe that all omissions would be catered for orally.

The terminology used was somewhat confusing and ambiguous. For example, the use of the surgical term "tumour" when describing an enlarged prostate is tantamount to telling a large proportion of the lay public that they will develop prostatic cancer. The use of conditional phrasing such as probably and may is also of limited use as some patients may be keen to obtain real figures from which to weigh up all possible risks and side effects associated with treatment.

Printed information about treatment from doctors is important to patients and so it needs to be relevant and easily understandable. Most surgeons do not have the time or resources to create and test the effectiveness of such printed information. If printed information on prostate and other surgery is to fulfil a greater role, improvements in terms of standardisation, form, and content will be required. This entails composition, testing on patients, graphics, and good printing, which require a range of nonsurgical skills outside the medical profession. There is a strong demand for information from patients, ${ }^{1-3}$ and it is important to fulfil this demand with good quality information produced with the help of people who experience the treatment - the patients. The Royal College of Surgeons of England is involved in producing information booklets for patients using patient feedback with professional presentation techniques and illustrations. ${ }^{7}$ Members of the National Prostatectomy Audit Group are: Mark
Emberton, David E Neal, Nick Black, Mark Fordham, Mark Harrison, Michael P McBrien, Robert E Williams, Klim McPherson, H Brendan Devlin. The national prostatectomy audit was funded by a grant from the Department of Health to Surgical Audit Unit of the Royal College of Surgeons of England and was conducted under the auspices of the British Association of Urological Surgeons through its audit working party, the Association of Surgeons of Great Britain and Ireland and the Health Services Research Unit at the London School of Hygiene and Tropical Medicine.

1 Wallace L. Informed consent to elective surgery: the therapeutic value? Soc Sci Med 1986;22:29-3

Meredith P. Patient participation in decision making and consent to treatment: the case of general surgery. Sociolog of Health and Illness 1993;15:115-36.

3 Williams O. Patient knowledge of operative care. $7 R$ Soc Med 1993;86:328-31. 
4 Mayberry JF, Rose J, Rhodes J. Assessment of a patient information booklet on ulcerative colitis. Ital f Gastroenterol 1989;21:193-5.

5 Audit Commission. What seems to be the matter? Communication between hospitals and patients. London: HMSO, 1993.
6 Emberton M, Neal D, Black N. Fordham M, Harrison $M$, McBrien MP, et al. The national prostatectomy audit: the clinical management of patients during hospital admission. Brf Urol (in press)

7 Devlin HB, ed. Surgery on the prostate: questions and answers. London: Royal College of Surgeons of England, 1994. 